\title{
Fail-safe truss topology optimization
}

\section{Stolpe, Mathias}

\section{Published in:}

Structural and Multidisciplinary Optimization

Link to article, DOI:

10.1007/s00158-019-02295-7

Publication date:

2019

\section{Document Version}

Peer reviewed version

Link back to DTU Orbit

\section{Citation (APA):}

Stolpe, M. (2019). Fail-safe truss topology optimization. Structural and Multidisciplinary Optimization, 60(4), 1605-1618. https://doi.org/10.1007/s00158-019-02295-7

\section{General rights}

Copyright and moral rights for the publications made accessible in the public portal are retained by the authors and/or other copyright owners and it is a condition of accessing publications that users recognise and abide by the legal requirements associated with these rights.

- Users may download and print one copy of any publication from the public portal for the purpose of private study or research.

- You may not further distribute the material or use it for any profit-making activity or commercial gain

- You may freely distribute the URL identifying the publication in the public portal

If you believe that this document breaches copyright please contact us providing details, and we will remove access to the work immediately and investigate your claim 


\title{
Fail-safe truss topology optimization
}

\author{
Mathias Stolpe ${ }^{1}$
}

Received: 14 November 2018 / Revised: 12 April 2019 / Accepted: 16 April 2019

(C) Springer-Verlag GmbH Germany, part of Springer Nature 2019

\begin{abstract}
The classical minimum compliance problem for truss topology optimization is generalized to accommodate for fail-safe requirements. Failure is modeled as either a complete damage of some predefined number of members or by degradation of the member areas. The considered problem is modeled as convex conic optimization problems by enumerating all possible damage scenarios. This results in problems with a generally large number of variables and constraints. A working-set algorithm based on solving a sequence of convex relaxations is proposed. The relaxations are obtained by temporarily removing most of the complicating constraints. Some of the violated constraints are re-introduced, the relaxation is resolved, and the process is repeated. The problems and the associated algorithm are applied to optimal design of two-dimensional truss structures revealing several properties of both the algorithm and the optimal designs. The working-set approach requires only a few relaxations to be solved for the considered examples. The numerical results indicate that the optimal topology can change significantly even if the damage is not severe.
\end{abstract}

Keywords Fail-safe optimal design · Truss topology optimization · Minimum compliance $\cdot$ Semidefinite programming · Second-order cone programming

\section{Introduction}

In fail-safe structural optimization, the resulting design is safe even after certain predefined types of damage conditions apply, i.e., the losses of structural properties due to failures can be mitigated. This property is inherently important to ensure safety. It can also be used to reduce the economic consequences of the damage by allowing the structure to continue operating, perhaps at lower loads, until the damage can either be repaired or the damaged part replaced. Fail-safe truss optimization can be applied for conceptual optimal design in all areas where the truss assumption is valid. It is also suitable for both educational purposes and for establishing benchmark problems.

One of the first articles on fail-safe structural truss sizing optimization dates back to the mid-1970s in Sun et al. (1976). The problems that are modeled and solved

Responsible Editor: Ming Zhou

Mathias Stolpe

matst@dtu.dk

1 DTU Wind Energy, Technical University of Denmark (DTU), Fredriksborgvej 399, 4000 Roskilde, Denmark in Sun et al. (1976) are minimum weight problems with constraints on local stresses, nodal displacement, and the lowest eigenfrequency. The numerical examples exclusively consider truss structures. In Sun et al. (1976), both the number and the locations of the damaged members are considered to be known a priori. The numerical examples are either small enough such that all combinations of damages can be enumerated or a small set of representative members are chosen as damaged. The former approach is used for optimal design of trusses with three and four members, whereas the latter approach is used for optimal fail-safe design of a three-dimensional 72-bar truss.

In Achtziger and Bendsøe (1999), problem formulations and theory for truss topology optimization intended to maximize the stiffness (minimize the compliance) of a degraded structure are presented. The degradation is modeled by reduction of the modulus of elasticity in each of the members by a continuous design variable. One of the main results from Achtziger and Bendsøe (1999) is that the topology for the considered problem does not change compared to the optimal topology of the classical minimum compliance problem.

In Mohr et al. (2014), models for topology optimization of truss structures for combined redundancy and robustness properties are proposed. The robustness part is modeled 
by perturbations to the external loads. The redundancy requirements are modeled by vanishing members. The considered problems are minimum mass problems with stress constraints modeled using members forces. The approach is based on a plastic design in which the crosssectional area variables are accompanied by variables representing member forces. The nominal design problem is thus a classical linear programming formulation for truss optimization, see, e.g., Dorn et al. (1964), Hemp (1973), Achtziger (1996), and Gilbert and Tyas (2003), among many others. The combined redundant and robust design problems, therefore, become mixed integer linear programs (MILP). The numerical experiments in Mohr et al. (2014) include examples of both two- and three-dimensional truss structures with up to 500 members in the ground structures.

The article (Kanno 2017) proposes problems and an algorithm for fail-safe structural optimization of trusses by considering the worst-case scenario of structural degradation. The degradation is modeled by a predefined number of bars completely loosing load carrying capacity. The structural performance is measured by solving a worstcase limit load factor problem similar to plastic design with stress constraints. The limit load problem is modeled and solved via a mixed integer linear programming. The optimal design problem is treated by a derivative-free sequential quadratic programming (SQP) method where stencil gradient approximations are used, and thus designs satisfying local optimality conditions are expected. The numerical experiments contain single-load truss examples with up to 19 bars in the ground structure and up to 2 damaged bars.

Fail-safe design using topology optimization of continuum structures was presented in Jansen et al. (2014). The article contains a statement of a minimum compliance problem with a volume constraint. The local failure of the structure is modeled by removing material in patches of predefined location, shape, and size. The objective function is modeled as the min-max of the compliance values over failure of the different domains. The authors approximate the max function by the Kreisselmeier-Steinhauser function (Kreisselmeier and Steinhauser 1983) and solve the problem using the method of moving asymptotes (Svanberg 1987). The work in Jansen et al. (2014) was generalized in the article (Zhou and Fleury 2016) where the positioning of the patches was more refined. In both articles the same main ideas as presented in Sun et al. (1976) were used, i.e., the location of the degradation was known and the corresponding areas or volumes were removed entirely.

In this manuscript, a minimum compliance truss sizing and topology design problem with a volume constraint is considered. This problem is in many aspects simpler compared to the problems modeled and solved in, e.g., Sun et al. (1976). The choice of focusing on the minimum compliance of trusses is of course the associated convexity properties (see, e.g., Bendsøe and Sigmund (2003)). The problems solved in Sun et al. (1976) are generally nonconvex due to the displacement and stress constraints. They additionally have issues with satisfying standard constraint qualifications due to the stress constraints. This is normally referred to as the singularity problem, see, e.g., Sved and Ginos (1968), Kirsch (1990), Rozvany (1996), Rozvany (2001), Cheng and Guo (1997), and Achtziger and Kanzow (2008). Due to these issues, it is therefore not certain that the global optimal fail-safe design is found with guarantee when considering the more engineering relevant problems. When considering minimum compliance, truss topology design problems global optimality can be assured due to convexity properties. The solved problems and resulting designs can therefore be used as benchmarks.

One of the major computational difficulties with fail-safe structural optimization problems is the inherent number of constraints required if the damage locations are unknown. Previous approaches have mostly used constraint aggregation techniques or restricted the number of constraints by assumptions. Common aggregation techniques will change the problem class for the considered problems, and thus potentially disable the use of robust and efficient special purpose optimization methods. In this work, we pursue another possibility which is based on a constraint generation approach. It is similar to the working-set approach developed in Verbart and Stolpe (2018) for sizing optimization of frame structures subjected to time-dependent loads and local strength constraints. Rather than solving a sequence of non-convex problems as in Verbart and Stolpe (2018), the proposed algorithm is based on solving a sequence of convex problems of increasing size by selecting a number of relevant constraints to include. The problems are all conic and convex and can be solved by efficient interior-point methods. This approach is demonstrated on truss topology optimization problems but could just as well be applied to design of other types of structures.

The manuscript is outlined as follows. First, an illustrative small-scale example shows that fail-safe optimal design problems in general have different properties compared to standard minimum compliance truss topology optimization problems (henceforth, denoted the nominal problem). The example also illustrates possible differences in the actual optimal topology between the two types of problems. The starting point for the optimal design problems is the modeling of the damage scenarios both for complete damage of members and degradation of the member areas. Based on the damage modeling, a nominal maximum stiffness problem formulation is followed by the statement of the corresponding fail-safe problem. The fail-safe problem is then reformulated as conic convex problems with generally many linear and conic constraints. A working-set algorithm, 
based on solving a sequence of convex relaxations, is proposed to deal with the many constraints. The behavior of the algorithm is studied by performing numerical experiments on two-dimensional truss topology optimization problems. Based on the problem formulations and the results from the numerical experiments, a set of conclusions are stated together with an outline of potential future research topics. The manuscript, finally, contains short listings of the main parts of the code used in the numerical experiments.

\subsection{Notation}

The notation $\mathbf{A} \succeq \mathbf{B}$, where $\mathbf{A}$ and $\mathbf{B}$ are symmetric matrices, means that $\mathbf{A}-\mathbf{B}$ is positive semidefinite. The notation $\|\mathbf{x}\|$ denotes the Euclidean norm of the vector $\mathbf{x}$ throughout. The minimum eigenvalue of the symmetric matrix $\mathbf{A}$ is denoted $\lambda_{\min }(\mathbf{A})$.

\subsection{A motivating three-bar truss example}

Consider the three-bar truss shown in Fig. 1 with the single external load $\mathbf{f}=\left(\begin{array}{ll}1 & 0\end{array}\right)^{T}$. An essentially identical example is presented in Zhou and Fleury (2016). The lower three nodes in the ground structure are fixed to a rigid wall. This is, in Fig. 1a and forthcoming figures, indicated by the filled circles (nodes). The bars are numbered from left to right and the bar lengths are $l_{1}=l_{3}=\sqrt{2}$ and $l_{2}=1$. The modulus of elasticity is set to $E=1$ for all bars. The design variables are chosen as the crosssectional areas of the three bars in the ground structure. The objective function is chosen to be the compliance while the constraints include lower and upper bounds on the design variables and a constraint limiting the total volume of the structure. Let $W$ denote the set of design variables satisfying these constraints. This ground structure has a relatively small amount of potential redundancy, and it is used to illustrate some of the optimization difficulties that can occur in fail-safe structural optimization. The lower bounds on the design variables are set to zero and the upper bounds to one. The volume limit $V$ is set to $V=\sqrt{2}$. For this data, the optimal solution to the nominal problem, i.e., the problem not considering damage of members, suggests that both bars 1 and 3 should have an area equal to one-half. This design is illustrated in Fig. 1b. This is also verified by numerically solving the problem. Imagine now that three damage scenarios are considered, each consisting of the removal of one of the three members. Let $\mathcal{J}_{\alpha}$ denote the index set of members which are removed in damage condition $\alpha$. In this situation, the possible damage scenarios are represented by $\mathcal{J}_{1}=\{1\}, \mathcal{J}_{2}=\{2\}$, and $\mathcal{J}_{3}=\{3\}$. Under standard technical assumptions, it can be ensured that for each damage condition, there is a design, represented by the cross-sectional areas in $\mathbf{v}$, satisfying the variable

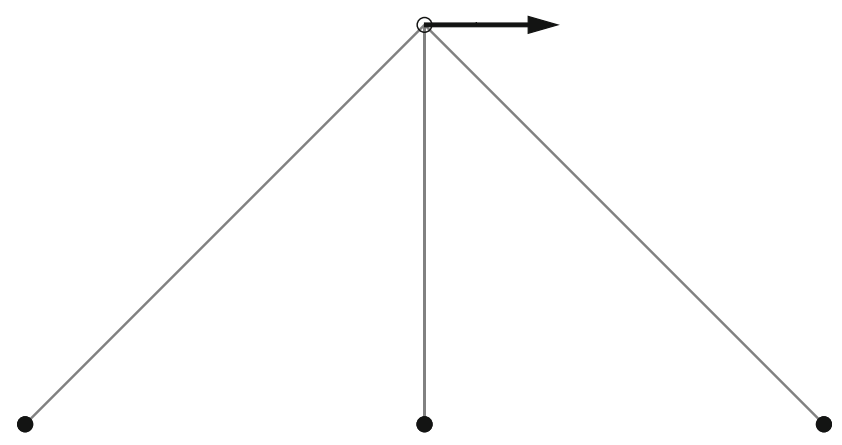

(a) Ground structure.

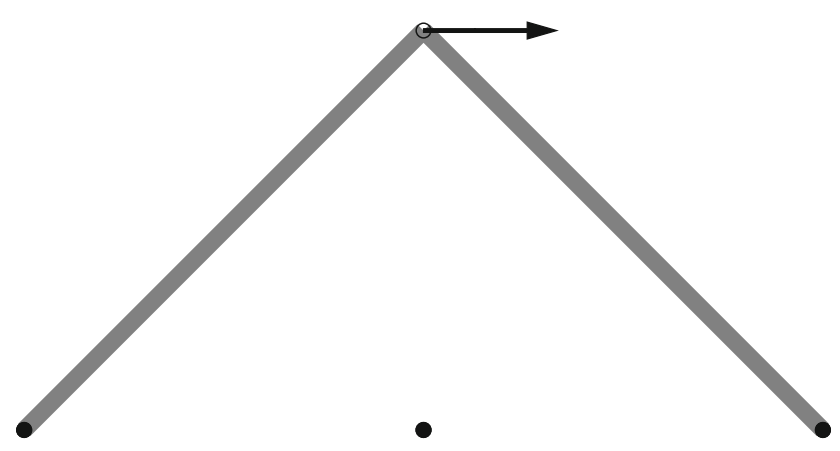

(b) Optimal nominal design.

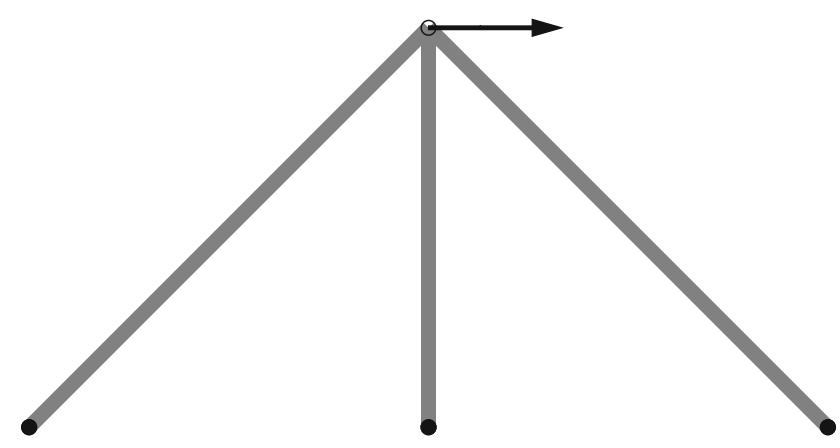

(c) Optimal fail-safe design.

Fig. 1 a A three-bar truss example with the objective to minimize compliance under a volume constraint and box constraints on the design variables. The compliance for the nominal design in $\mathbf{b}$ is $\approx 2.8284$, whereas the compliance for the fail-safe design in $\mathbf{c}$ is $\approx 3.8284$

bounds and the volume constraint, and at the same time ensures that $\mathbf{f} \in \operatorname{range}\left(\mathbf{K}^{\alpha}(\mathbf{v})\right)$ for all $\alpha$. Here, and in the rest of the manuscript, $\mathbf{K}^{\alpha}(\mathbf{v})$ is the assembled stiffness matrix modeling the partially or completely damaged members listed in the index-set $\mathcal{J}_{\alpha}$. The solution to the corresponding fail-safe problem consists of three bars as illustrated in Fig. 1c. This type of design is also found in Zhou and Fleury (2016). The numerical results suggest that all bars have the same area at the optimum. It is also verified numerically, at least up to the eleventh digit, that the optimal area is $\sqrt{2} /(2 \sqrt{2}+1)$. The compliance for the nominal design is 
$2 \sqrt{2}$ and the compliance for the fail-safe design is $1+2 \sqrt{2}$. This is an increase of more than $35 \%$ in the objective function. If, on the other hand, the damage conditions of the model simultaneously remove two bars, then the situation changes abruptly. If the set $\mathcal{J}_{1}=\{1,3\}$ is chosen, then the damaged structure has no stiffness in the $x$-direction independently of how the design variables are chosen, i.e., $\mathbf{f} \notin \operatorname{range}\left(\mathbf{K}^{\alpha}(\mathbf{v})\right)$ for any $\mathbf{v} \in W$. The situation is similar for the other damage scenarios and the fail-safe problem lacks feasible solutions.

\section{Structural and damage modeling}

This section presents how the stiffness matrix $\mathbf{K}(\mathbf{v})$ is modeled and computed for both the undamaged structure (the nominal structure) and for the case of the simple damage models which are considered. The model closely follows the member degradation models used for worstcase detections in a limit analysis presented in Kanno (2012). The model is also closely related to the concept of strong redundancy presented in Kanno and Ben-Haim (2011). The computation of the nominal stiffness matrix is described by a standard assembly process for truss structures parametrized by the cross-sectional member areas. In the following, damage condition $\alpha$ denotes a specific combination of members that are either completely damaged or degraded.

\subsection{Complete damage of members}

Let $\mathcal{N}=\{1, \ldots, n\}$ denote the set of members in the structure. Furthermore, let $\mathcal{J}_{\alpha} \subseteq \mathcal{N}$ denote the index set of members which are removed or degraded in damage scenario $\alpha$. The stiffness matrix for the nominal (undamaged) truss structure is linear in the design variables, i.e., the member cross-sectional areas $\mathbf{v}$, and given by the following:

$\mathbf{K}(\mathbf{v})=\sum_{j \in \mathcal{N}} v_{j} \mathbf{K}_{j}$

where $\mathbf{K}_{j} \in \mathbb{R}^{d \times d}$ is the scaled element stiffness matrix for the $j$ th bar and $d$ is the number of degrees of freedom after removal of fixed degrees of freedom. The element stiffness matrices are given by the following:

$\mathbf{K}_{j}=\frac{E}{l_{j}} \mathbf{r}_{j} \mathbf{r}_{j}^{T}$

where $E>0$ is the Young's modulus of the chosen material, $\mathbf{r}_{j} \in \mathbb{R}^{d}$ contains the direction cosines, and $l_{j}>0$ is the length of the $j$ th bar, respectively. The structural analysis of the damaged structure requires modifications of the stiffness matrix. For damage condition $\alpha$ and in the case of complete removal of the members listed in $\mathcal{J}_{\alpha}$, the stiffness matrix becomes as follows:

$\mathbf{K}^{\alpha}(\mathbf{v})=\sum_{j \in \mathcal{N} \backslash \mathcal{J}_{\alpha}} v_{j} \mathbf{K}_{j}$

Note that design sensitivity analysis of the stiffness matrix is readily available analytically for both the nominal structure and the damaged structures.

\subsection{Degradation of members}

In the case of partial damage (degradation), the member is not removed entirely from the stiffness matrix computations. Instead, the situation is modeled by an area reduction of the members listed in the set $\mathcal{J}_{\alpha}$ leading to a reduction in the stiffness of the structure. In this situation, the stiffness matrix is modeled by the following:

$\mathbf{K}^{\alpha}(\mathbf{v})=\sum_{j \in \mathcal{N} \backslash \mathcal{J}_{\alpha}} v_{j} \mathbf{K}_{j}+\sum_{j \in \mathcal{J}_{\alpha}} r\left(v_{j}\right) \mathbf{K}_{j}$

where $r(v)$ is a function assumed to satisfy the following requirements:

- $\quad r(v)$ is linear in $v$ and $0 \leq r(v) \leq v$ for all $v \geq 0$.

These assumptions ensure that the computed area is nonnegative and that the computed area is smaller than the nominal area. When $r(v)$ is linear in $v$, then several convex reformulations are possible.

\section{Structural optimization problems}

The nominal worst-case minimum compliance truss topology design problem is first presented together with natural assumptions collected from the literature. The fail-safe version of the nominal problem is then modeled as minimization of the worst-case compliance computed over all damage scenarios.

\subsection{Nominal problem}

The nominal worst-case multiple load minimum compliance problem is following, e.g., Achtziger (1998) stated as follows:

$$
\begin{array}{ll}
\underset{\mathbf{v}, \mathbf{u}}{\operatorname{minimize}} & \max _{1 \leq \ell \leq L}\left\{\mathbf{f}_{\ell}^{T} \mathbf{u}_{\ell}\right\} \\
\text { subject to } & \mathbf{K}(\mathbf{v}) \mathbf{u}_{\ell}-\mathbf{f}_{\ell}=\mathbf{0} \quad \ell=1, \ldots, L \\
& \sum_{j \in \mathcal{N}} v_{j} l_{j}<V \\
& \mathbf{v}^{\min } \leq \mathbf{v} \leq \mathbf{v}^{\max }
\end{array}
$$

Here, $L$ is the number of external static loads, $V>0$ is a given bound on the structural volume, and $\mathbf{v}^{\mathrm{min}}$ and 
$\mathbf{v}^{\max }$ are given lower and upper bounds on the design variables. The vector $\mathbf{u}=\left(\mathbf{u}_{1}^{T} \cdots \mathbf{u}_{L}^{T}\right)^{T}$ contains the displacement vectors for all load cases. Following Achtziger et al. (1992) and Achtziger (1998), we assume throughout that the problem data satisfies the following conditions:

- $\mathbf{f}_{\ell} \neq 0$ for $\ell=1, \ldots, L$,

$-0 \leq \mathbf{v}^{\min }<\mathbf{v}^{\max }$,

- $\quad \sum_{j \in \mathcal{N}} v_{j}^{\min } l_{j}<V<\sum_{j \in \mathcal{N}} v_{j}^{\max } l_{j}$, and

- $\quad \mathbf{K}(\mathbf{v})$ is positive definite for all $\mathbf{v}>0$.

The minimum compliance truss design problem $\left(\mathrm{P}_{\mathrm{N}}\right)$ and similar problems have been studied in numerous articles and books (see, e.g., the detailed theoretical treatment in Achtziger (1998) and the textbook (Bendsøe and Sigmund 2003)).

The set $W$ is introduced to model the variable bounds and the volume constraint, i.e.,

$W=\left\{\mathbf{v} \in \mathbb{R}^{n} \mid \mathbf{v}^{\min } \leq \mathbf{v} \leq \mathbf{v}^{\max }, \sum_{j \in \mathcal{N}} v_{j} l_{j} \leq V\right\}$.

Due to the above stated assumptions, it is ensured that $W \neq \emptyset$. If these assumptions are imposed on the problem data, then the minimum compliance problem $\left(\mathrm{P}_{\mathrm{N}}\right)$ has a non-empty feasible set and optimal solutions are attained (see, Achtziger (1998)).

An alternative to the worst-case objective function is to consider the weighted average minimum compliance problem, i.e., to replace the objective function in $\left(\mathrm{P}_{\mathrm{N}}\right)$ by the following:

$\sum_{\ell=1}^{L} \delta_{l} \mathbf{f}_{\ell}^{T} \mathbf{u}_{\ell}$

where $\delta_{\ell} \geq 0$ are given constants additionally satisfying $\sum_{\ell} \delta_{\ell}=1$. This change to the objective function, however, gives only minor changes to the fail-safe problem formulations, and this approach is therefore not covered in any additional detail.

\subsection{Fail-safe problem and SDP reformulation}

The fail-safe equivalent to the minimum compliance problem $\left(\mathrm{P}_{\mathrm{N}}\right)$ becomes a standard nonlinear optimization problem when the locations of the damages are either known or can be enumerated. In both situations, the problem can be formulated as follows:

$$
\begin{aligned}
& \operatorname{minimize} \tau \\
& \tau \geq 0, \mathbf{v} \in W, \mathbf{u} \\
& \text { subject to } \mathbf{K}^{\alpha}(\mathbf{v}) \mathbf{u}_{\ell}^{\alpha}-\mathbf{f}_{\ell}=\mathbf{0} \forall \ell, \alpha=1, \ldots, p \\
& \mathbf{f}_{\ell}^{T} \mathbf{u}_{\ell}^{\alpha} \leq \tau, \quad \forall \ell, \alpha=1, \ldots, p
\end{aligned}
$$

where $p$ is the total number of damage scenarios. In problem $(\mathrm{P})$, it is assumed that the external loads do not change. This can, to some extent, be incorporated by introducing an index $\alpha$ also for the loads. One could imagine that there is an additional control system in the structure which can mitigate the loads in case of damage. Problem (P) can equivalently be reformulated as a convex problem in a number of ways. One such reformulation results in the semidefinite program (SDP) is as follows:

$$
\begin{aligned}
& \underset{\tau \geq 0, \mathbf{v} \in W}{\operatorname{minimize}} \tau \\
& \text { subject to }\left(\begin{array}{ll}
\tau & \mathbf{f}_{\ell}^{T} \\
\mathbf{f}_{\ell} & \mathbf{K}^{\alpha}(\mathbf{v})
\end{array}\right) \succeq \mathbf{0} \forall(\ell, \alpha)
\end{aligned}
$$

This kind of reformulation is proposed in numerous places in the literature (see, e.g., Ben-Tal and Nemirovski (1997), Ben-Tal and Nemirovski (2001), and Achtziger and Kočvara (2007)), for nominal minimum compliance problems. Note that this problem is similar in its mathematical structure to a standard problem with many load cases. The obvious, but important difference is that the load is identical for all damage scenarios and that the stiffness matrix changes slightly. Problem $\left(\mathrm{P}_{\mathrm{SDP}}\right)$ has a potentially very large number of matrix inequalities but a reduced set of variables compared to, e.g., (P) due to the removal of the state variables. Convexity of $\left(\mathrm{P}_{\mathrm{SDP}}\right)$ follows immediately since the objective function is linear, the matrix inequalities are linear in the variables and the rest of the feasible set is defined by linear inequalities (see, e.g., Boyd and Vandenberghe (2004)). The lower bound $\tau \geq 0$ is redundant and could be omitted from problem ( $\left.\mathrm{P}_{\mathrm{SDP}}\right)$ and the following problems since compliance is always positive. Preliminary numerical results suggest that the chosen solvers often require fewer iterations and produce more accurate results if this constraint is included. It is therefore included both in the theoretical developments and in the code used to generate the numerical results.

For these minimum compliance problems and the choice of damage/degradation models, it is not necessary to include damage scenarios involving fewer bars than considered. This is because if there are two design vectors $\mathbf{v}^{1}$ and $\mathbf{v}^{2}$ satisfying $\mathbf{v}^{1} \leq \mathbf{v}^{2}$, then $\mathbf{f}^{T} \mathbf{u}^{1} \geq \mathbf{f}^{T} \mathbf{u}^{2}$ where the displacement vectors $\mathbf{u}^{1}$ and $\mathbf{u}^{2}$ satisfy the equilibrium equations $\mathbf{K}\left(\mathbf{v}^{i}\right) \mathbf{u}^{i}=\mathbf{f}$. The motivation for this result is that the partial derivatives of compliance are always non-positive (see, e.g., Bendsøe and Sigmund (2003)) for the considered model of the stiffness matrix. A damage scenario involving $k$ bars is thus conservative compared to damage scenarios with $k-1$ bars.

\subsection{Fail-safe problem and SOCP reformulation}

An alternative to the SDP problem ( $\left.\mathrm{P}_{\mathrm{SDP}}\right)$ is to reformulate (P) as a second-order cone program (SOCP) (see, e.g., Lobo 
et al. (1998)). The proposed reformulation is very similar to those proposed for minimum compliance problems in, e.g., Ben-Tal and Nemirovski (2001) and Kanno (2016). In SOCP reformulations of minimum compliance problems, the members forces $q_{j}$ are included as variables. The SOCP reformulation is based on the following arguments which for simplicity are mainly presented without the load indices $\ell$. The area reduction is modeled by $r\left(v_{j}\right)=(1-\gamma) v_{j}$ for some constant $\gamma \in[0,1]$, i.e., the relative damage. If $\gamma=0$, the nominal problem is retrieved and if $\gamma=1$ complete damage of members is modeled. Node equilibrium of forces is written as the linear equality constraints as follows:

$\sum_{j \in \mathcal{N} \backslash \mathcal{J}_{\alpha}} q_{j} \mathbf{r}_{j}+\sum_{j \in \mathcal{J}_{\alpha}}(1-\gamma) q_{j} \mathbf{r}_{j}=\mathbf{f}$.

The compliance is written as follows:

$$
\begin{aligned}
\mathbf{u}^{T} \mathbf{K}(\mathbf{v}) \mathbf{u} & =\sum_{j \in \mathcal{N} \backslash \mathcal{J}_{\alpha}} v_{j} \frac{E}{l_{j}}\left(\mathbf{r}_{j}^{T} \mathbf{u}\right)^{2}+\sum_{j \in \mathcal{J}_{\alpha}}(1-\gamma) v_{j} \frac{E}{l_{j}}\left(\mathbf{r}_{j}^{T} \mathbf{u}\right)^{2} \\
& =\sum_{j \in \mathcal{N} \backslash \mathcal{J}_{\alpha}} \frac{l_{j}}{E} \frac{q_{j}^{2}}{v_{j}}+\sum_{j \in \mathcal{J}_{\alpha}}(1-\gamma) \frac{l_{j}}{E} \frac{q_{j}^{2}}{v_{j}}
\end{aligned}
$$

with the interpretation that $q_{j}^{2} / v_{j}=0$ when the crosssectional area vanishes. After introducing the additional variables, $\eta_{j} \geq 0$ and the inequalities are as follows:

$\frac{q_{j}^{2}}{v_{j}} \leq \eta_{j}$ for all $j \in \mathcal{N}$

the compliance constraint $\mathbf{f}^{T} \mathbf{u}=\mathbf{u}^{T} \mathbf{K}(\mathbf{v}) \mathbf{u} \leq \tau$ is written as follows:

$\sum_{j \in \mathcal{N} \backslash \mathcal{J}_{\alpha}} \frac{l_{j}}{E} \eta_{j}+\sum_{j \mathcal{J}_{\alpha}}(1-\gamma) \frac{l_{j}}{E} \eta_{j} \leq \tau$.

The final step of the reformulation is based on a result regarding hyperbolic constraints from, e.g., Lobo et al. (1998).

$w^{2} \leq x y, x \geq 0, y \geq 0 \Longleftrightarrow\left\|\left(\begin{array}{c}2 w \\ x-y\end{array}\right)\right\| \leq x+y$.

The SOCP formulation of the fail-safe minimum compliance problem for the complete damage model becomes as follows:

$$
\begin{array}{ll}
\underset{\tau, \mathbf{v} \in W, \mathbf{q}, \eta}{\operatorname{minimize}} \tau & \\
\text { subject to } \sum_{j \in \mathcal{N} \backslash \mathcal{J}_{\alpha}} q_{j \ell}^{\alpha} \mathbf{r}_{j}+\sum_{j \in \mathcal{J}_{\alpha}}(1-\gamma) q_{j \ell}^{\alpha} \mathbf{r}_{j}=\mathbf{f}_{\ell} & \forall(\ell, \alpha) \\
\sum_{j \in \mathcal{N} \backslash \mathcal{J}_{\alpha}} \frac{l_{j}}{E} \eta_{j \ell}^{\alpha}+\sum_{j \in \mathcal{J}_{\alpha}}(1-\gamma) \frac{l_{j}}{E} \eta_{j \ell}^{\alpha} \leq \tau & \forall(\ell, \alpha) \\
\left\|\left(2 q_{j \ell}^{\alpha} \eta_{j \ell}^{\alpha}-v_{j}\right)^{T}\right\| \leq \eta_{j \ell}^{\alpha}+v_{j} & \forall(j, \ell, \alpha) \\
\tau \geq 0, \eta_{j \ell}^{\alpha} \geq 0 & \forall(j, \ell, \alpha)
\end{array}
$$

( $\left.\mathrm{P}_{\mathrm{SOCP}}\right)$
Problem $\left(\mathrm{P}_{\mathrm{SOCP}}\right)$ is also a convex conic optimization problem following standard arguments (see, e.g., Boyd and Vandenberghe (2004)). The problem has a potentially large number of both equality and inequality constraints together with the second-order cone constraints.

\section{A working-set algorithm}

For the fail-safe minimum compliance problem $(\mathrm{P})$, one could conjecture that only a small number of the damage scenarios are active at any optimal solution. This suggests that an algorithm based on solving a sequence of relaxations obtained by temporary removal of most constraints and subsequent re-introduction of relevant constraints could be computationally beneficial. This is under the assumptions that the number of design variables is modest compared to the number of damage scenarios. This is often the case if two (or more) members are simultaneously removed in the damage scenarios. Then, the number of scenarios becomes $n(n-1) / 2$ where $n$ is the number of members.

Given $(\tau, \mathbf{v}) \in \mathbb{R}_{+} \times W$ define the set of violated constraint for the SDP problem $\left(\mathrm{P}_{\mathrm{SDP}}\right)$, as follows:

$$
\begin{aligned}
\mathcal{V}(\tau, \mathbf{v})= & \{(\ell, \alpha) \mid \ell \in\{1, \ldots, L\}, \alpha \in\{1, \ldots, p\}, \\
& \left.\lambda_{\min }\left(\begin{array}{cc}
\tau & \mathbf{f}_{\ell}^{T} \\
\mathbf{f}_{\ell} & \mathbf{K}^{\alpha}(\mathbf{v})
\end{array}\right)<0\right\} .
\end{aligned}
$$

Introduce the notation $\mathcal{V}^{k}=\mathcal{V}\left(\tau^{k}, \mathbf{v}^{k}\right)$ for the set of violated constraints at iterate $k$.

The main idea in the working-set approach is to first solve the nominal design problem $\left(\mathrm{P}_{\mathrm{N}}\right)$ by some available convex reformulation and then compute the constraints for all damage scenarios and loads, and in the same process compute the set of violated matrix inequalities. The working set $\mathcal{W}^{k}$ is then updated to additionally include at least one of the constraints violated at the current point $\left(\tau^{k}, \mathbf{v}^{k}\right)$. The relaxed problem is then resolved and the process is repeated. The number of constraints listed in the working set, thus increases with at least one for every outer iteration. If the optimum for the relaxed problem is not obtained, then the optimum for the fail-safe problem (P) (or alternatively $\left(\mathrm{P}_{\mathrm{SDP}}\right)$ ) is not obtained and the objective function goes to infinity. The relaxation which is solved in every outer iteration of the working-set approach is for the SDP reformulation as follows:

$$
\begin{aligned}
& \underset{\tau \geq 0, \mathbf{v} \in W}{\operatorname{minimize}} \tau \\
& \text { subject to }\left(\begin{array}{ll}
\tau & \mathbf{f}_{\ell}^{T} \\
\mathbf{f}_{\ell} & \mathbf{K}^{\alpha}(\mathbf{v})
\end{array}\right) \succeq \mathbf{0}(\ell, \alpha) \in \mathcal{W}^{k}
\end{aligned}
$$$$
\left(\mathrm{P}_{\mathrm{SDP}}^{k}\right)
$$

A similar relaxation can be stated for the second-order cone problem $\left(\mathrm{P}_{\mathrm{SOCP}}\right)$. Let the set of critical constraints at the 
point $\left(\tau^{k}, \mathbf{v}^{k}\right)$ be denoted by $\mathcal{C}^{k} \subseteq \mathcal{V}^{k}$. This set contains a user defined list of constraints which contribute to the constraint violation measure and should be considered for inclusion in the working set. Then, the updated working set is given by the following:

$\mathcal{W}^{k+1}=\mathcal{W}^{k} \cup \mathcal{C}^{k}$

If $\mathcal{V}^{k}=\emptyset$, then there are no violated constraints and if the relaxation $\left(\mathrm{P}_{\mathrm{SDP}}^{k}\right)$ has been solved then the original problem (P) has also been solved. In this situation, the set of critical constraints $\mathcal{C}^{k}$ is also empty and the algorithm terminates. If, on the other hand, $\mathcal{V}^{k} \neq \emptyset$, then choose $\mathcal{C}^{k}$ such that $\left|\mathcal{C}^{k}\right| \geq 1$, i.e., at least one constraint is added to the working set. By construction of the relaxation $\mathcal{C}^{k} \cap \mathcal{V}^{k}=\emptyset$ and in this situation $\left|\mathcal{W}^{k+1}\right|>\left|\mathcal{W}^{k}\right|$. Since there are finitely many constraints, the working set can only be updated a finite number of times. The working-set approach is presented in detail in Algorithm 1. Since the relaxations such as $\left(\mathrm{P}_{\mathrm{SDP}}^{k}\right)$ are convex, they can be solved to global optimality, e.g., using an interior-point method specialized for SDP such as SDPA (Fujisawa et al. 1997) and Yamashita and Fujisawa (2003), SeDuMi (Sturm 1999) and Sturm (2002), or SDPT3 (Toh et al. 1999) and Tütüncü et al. (2003), among several others.

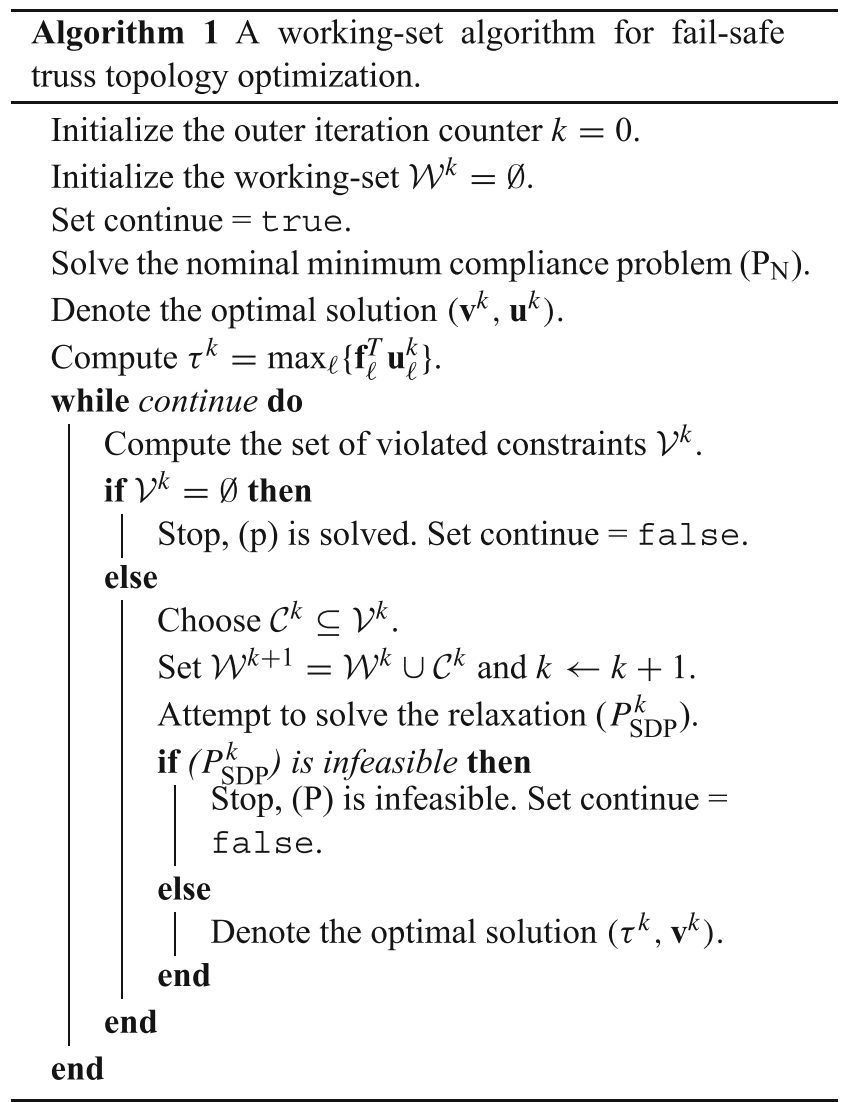

The numerical performance for this kind of algorithm is dependent on the choice of the set $\mathcal{C}^{k}$. One can, e.g., define a finitely convergent algorithm by adding one violated constraint at the time and not remove any constraints from the working set. This could result in many relaxations to be solved causing extensive computation times. Alternatively, the critical constraint set $\mathcal{C}^{k}$ could be chosen to include several, or even many, violated constraints. This could reduce the number of outer iterations but the computation time for each relaxation could increase substantially. Different parameter values for this choice are proposed and evaluated in the next section.

\section{Implementation}

The structural analysis and the optimization problem modeling are entirely implemented in Matlab version 2018a (The Mathworks Inc 2018). The optimization problems are specified and solved using the modeling system for convex optimization CVX (see, Grant and Boyd (2008) and Grant and Boyd (2014)). The critical parts of the code are presented in Appendix A for the nominal problems and in Appendix B for the failsafe relaxations, respectively. All convex optimization problems are numerically solved using SeDuMi (see, Sturm (1999) and Sturm (2002)). The working set is also used together with SDPT3 as the optimization solver too, and this gives the same overall behavior and final results. Default parameters are used in the modeling and the optimization solvers with the exception that the precision flag is set to high in CVX (cvx_precision high), and this information is passed to the solver. The problem data, i.e., the external load, material properties, and the geometry, is scaled in the computations such that the convex optimization problems can be numerically solved to high accuracy.

In the implementation of the working-set approach and the numerical experiments, the choices of problem scaling by changing $E$ and the external load $\mathbf{f}$ and the various tolerances (feasibility and optimality) become important. It is necessary to appropriately balance the tolerance used to determine if a constraint is active or violated to the feasibility and optimality tolerances used in the optimization solvers.

In the implementation, feasibility of the stiffness constraints modeled as linear matrix inequalities is determined by computing all eigenvalues of the matrices:

$$
\left(\begin{array}{ll}
\tau^{k} & \mathbf{f}_{\ell}^{T} \\
\mathbf{f}_{\ell} & \mathbf{K}^{\alpha}\left(\mathbf{v}^{k}\right)
\end{array}\right)
$$

This is done by storing the matrices as dense and using the dense eigenvalue routines that are part of Matlab. The 
dense storage format is motivated by the relatively few degrees of freedom of the considered problems. Alternative storage and algorithm choices could perform better for larger systems. A matrix inequality is considered violated if the minimum eigenvalue is less than $-\epsilon$ where the feasibility tolerance $\epsilon$ is set to $10^{-9}$.

\section{Numerical experiments}

In the numerical experiments, several design domains are considered. The lower bounds on the design variables are set to zero and the upper bounds to one for all examples. For all examples, the Young's modulus is set to $E=100$ since this value combined with the chosen external loads result in well-scaled problem instances that can be robustly and accurately solved.

The first set of examples are based on a cantilever beam with dimensions $6 \times 1$ and a vertical load applied at the lower right corner. The nodes on the left-hand side are all fixed to a rigid wall. The ground structure consists of $15 \times 3$ equidistant nodes and 128 members. The volume limit $V$ is chosen to be $10 \%$ of the total possible volume.

The second set of examples are based on a shorter cantilever beam with dimensions $3 \times 1$. The ground structure consists of $4 \times 2$ equidistant nodes and in total 19 bars. The nodes on the left-hand side are again fixed to a rigid wall. A horizontal load is applied at the two nodes on the righthand side, each non-zero component if the force vector has a value of one. This example is identical to one from Kanno (2017) in terms of the supports, load, and ground structure. The volume limit $V$ is chosen to be $10 \%$ of the total possible volume.

The third set of examples is based on a design domain with dimensions $1 \times 1$. The ground structure consists of $9 \times 9$ equidistant nodes and 272 bars. The nodes are again fixed to a rigid wall on the left side and a unit vertical load is applied at lower right-hand node. The volume limit $V$ is chosen to be $5 \%$ of the total possible volume.

The considered ground structures for these three examples are shown in Fig. 2. None of the ground structures contain overlapping bars. For the ground structure of Examples I and III, only immediately neighboring nodes are connected. For Example II, some bars connect nodes further than to just being direct neighbors.

The fail-safe problem characteristics are presented in Table 1. In the damage column, both the type and the amount of damage is presented. Within parenthesis, the number of damaged bars is reported together with the relative amount of partial damage, i.e., the factor $\gamma$.

The numerical experiments are run on a laptop with an Intel Core i7-5600U processor running at $2.60 \mathrm{GHz}$ without the use of any parallel computations.

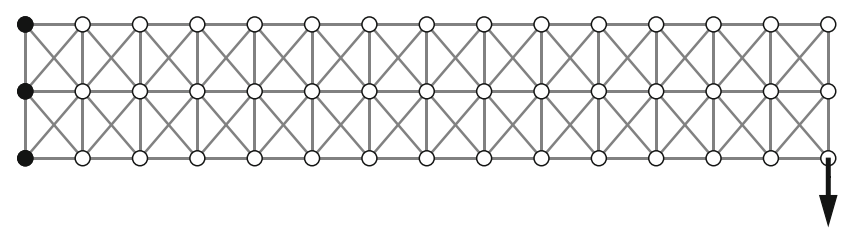

(a)

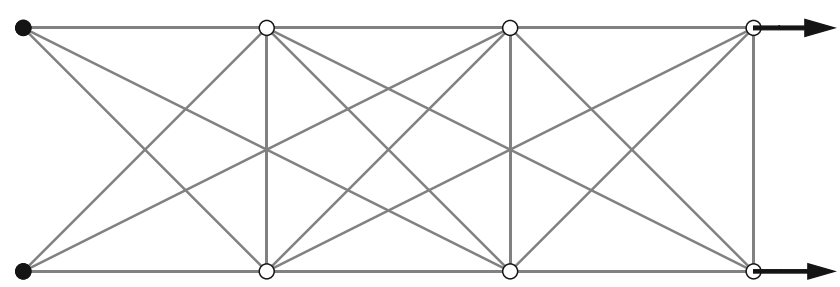

(b)

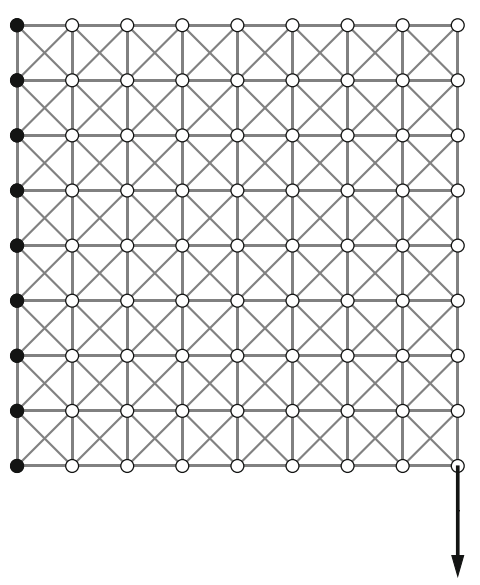

(c)

Fig. 2 Ground structures for the three truss topology optimization examples. a Example I with $n=128$ bars. b Example II with $n=19$ bars. c Example II with $n=272$ bars

Table 1 General ground structure and problem characteristics

\begin{tabular}{|c|c|c|c|c|c|}
\hline $\mathrm{P}$. & Damage & $N_{x} \times N_{y}$ & $d$ & $n$ & Scenarios \\
\hline I & Nominal $(-,-)$ & $15 \times 3$ & 84 & 128 & - \\
\hline I & Complete $(1,-)$ & $15 \times 3$ & 84 & 128 & 128 \\
\hline I & Degradation $(1, \gamma)$ & $15 \times 3$ & 84 & 128 & 128 \\
\hline I & Degradation $(2, \gamma)$ & $15 \times 3$ & 84 & 128 & 8128 \\
\hline II & Nominal $(-,-)$ & $4 \times 2$ & 12 & 19 & - \\
\hline II & Complete $(1,-)$ & $4 \times 2$ & 12 & 19 & 19 \\
\hline II & Complete $(2,-)$ & $4 \times 2$ & 12 & 19 & 171 \\
\hline II & Degradation $(1, \gamma)$ & $4 \times 2$ & 12 & 19 & 19 \\
\hline II & Degradation $(2, \gamma)$ & $4 \times 2$ & 12 & 19 & 171 \\
\hline III & Nominal $(-,-)$ & $9 \times 9$ & 144 & 272 & - \\
\hline III & Complete $(1,-)$ & $9 \times 9$ & 144 & 272 & 272 \\
\hline III & Degradation $(1, \gamma)$ & $9 \times 9$ & 144 & 272 & 272 \\
\hline III & Degradation $(2, \gamma)$ & $9 \times 9$ & 144 & 272 & 36856 \\
\hline
\end{tabular}




\subsection{Nominal designs}

Before presenting the fail-safe designs, the nominal problem $\left(\mathrm{P}_{\mathrm{N}}\right)$ is solved for all ground structures. The optimal designs are depicted in Fig. 3. In all figures of optimal designs, members with cross-sectional area smaller than $10^{-7} \max _{i}\left\{v_{i}^{\max }\right\}$ are excluded. This is because all the problems are solved using interior-point methods and some non-zero values are always present in the numerical results even for bars which should be removed. For Example I, a classical cantilever beam is found. For Example II, the optimal nominal design (essentially) consists of two disconnected bars. The optimal design is qualitatively identical to the one found in Kanno (2017). For Example III, a classical two-bar truss is obtained. The optimal designs for the nominal problems are all statically determinate (see, Fig. 3), and many of the bars in the ground structures have vanished, i.e., have a numerically very close to zero cross-sectional area. Since these three structures are statically determinate, there exists one bar whose

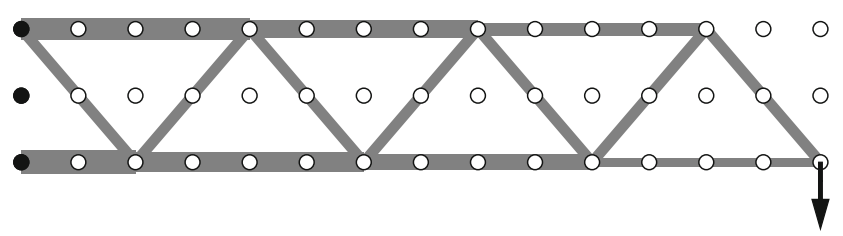

(a) Cantilever design for Example I.

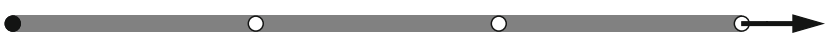

(b) Two disconnected bars for Example II.

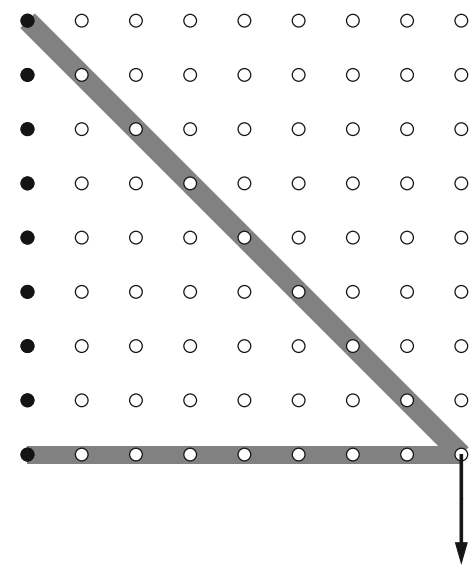

(c) Two-bar truss for Example III.

Fig. 3 Optimal structures for the nominal minimum compliance problems for a Example I, b Example II, and c Example III removal would make it impossible to satisfy the equilibrium conditions. These structures are therefore infeasible to failsafe problems in which one or more bars are removed.

\subsection{Complete damage examples}

In the first set of examples, we consider the situation that exactly one member completely loses the ability to carry load. Since the ground structures contain a fairly small number of members, it is possible to model and solve problems with $n$ matrix inequalities. This approach is also used to verify that the working-set approach produces the same solutions but this approach is not reported. The optimal designs for the three examples for this situation are depicted in Fig. 4.

Compared to the nominal designs, the fail-safe requirement results in complete changes of optimal topologies for all three examples as shown in Fig. 4. In the optimal failsafe designs, almost all members in the ground structure

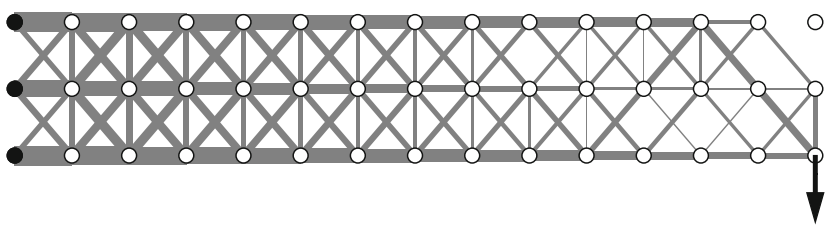

(a) Example I.

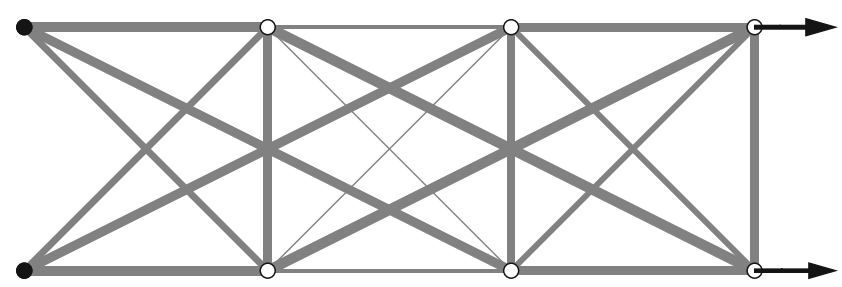

(b) Example II.

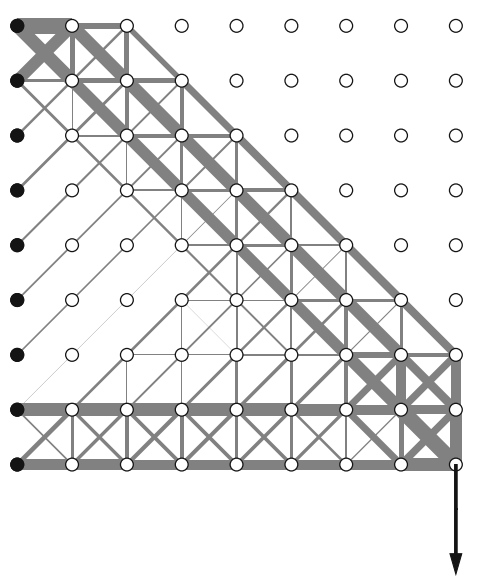

(c) Example III.

Fig. 4 Optimal structures for the fail-safe compliance problems with complete damage of exactly one member for a Example I, b Example II, and c Example III 
have non-zero areas. For Example II, the optimal fail-safe design is qualitatively identical to the corresponding design in Kanno (2017).

For the ground structures, for Examples I and III, as illustrated in Fig. 2a and c, one can observe that damage scenarios including removal of two (or more) bars will not result in feasible designs and problems with several complete damages are therefore not investigated numerically. This is similar to the situation outlined for the three-bar truss problem. This kind of infeasibility is caused by the truss assumption, i.e., the modeling of the joints. It will most likely not occur in more than pathological situations for, e.g., frame structures.

\subsection{Degradation examples}

In the next set of problems, the number of damaged members is increased to two and rather than completely removing some members the bar areas are reduced. If two bars are degraded, then the number of damage scenarios is $n(n-1) / 2$. Optimal fail-safe designs for Example I for several values of the relative damage parameter $\gamma$ are presented in Fig. 5.

The behavior of the active-set approaches is reported in Tables 2, 3, and 4 . In these tables, $m$ is the user-defined limit on the cardinality of the set of critical constraints, i.e., $\left|\mathcal{C}^{k}\right| \leq m$ for all $k$. The columns labeled Itn. in these tables refer to the number of solved minimum compliance problems including the nominal problem. The column with heading $|\mathcal{W}|$ shows the final number of load and damage scenarios in the final working set, and $|\mathcal{A}|$ is the actual number of active damage scenarios in the working set. Note that the total number of active constraints can be larger since the working set only incorporates constraints that at some point are violated, and may thus not include all active constraints, which from a computational point of view can be an advantage.

The timings reported in Tables 2, 3, and 4 include all parts of the process ranging from the ground structure generation, modeling and parsing of the problem, and the solver time. Preliminary numerical experiments suggest that the SDP formulations result in shorter computation times because of the parsing of the problems. The SOCP problems have significantly more constraints, and this is time-consuming. Another implementation could result in completely different observations.

The cantilever beam is used for choosing appropriate value on the maximum number of added constraints between two outer iterations, i.e., the upper limit $m$ on $\left|\mathcal{C}^{k}\right|$. As expected, Table 2 suggests that a too low value results in many relaxations to be solved. On the other hand, high a value can decrease the number of relaxations to be solved but can instead results in an overestimation

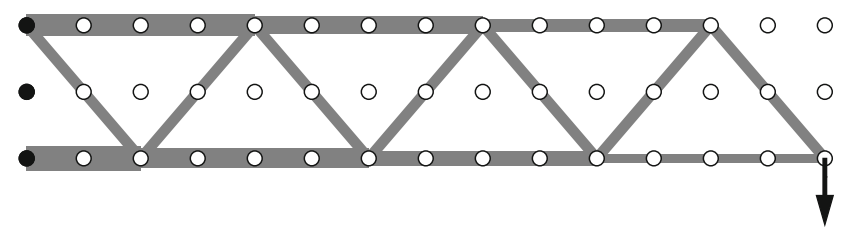

(a) $\gamma=0.7$.

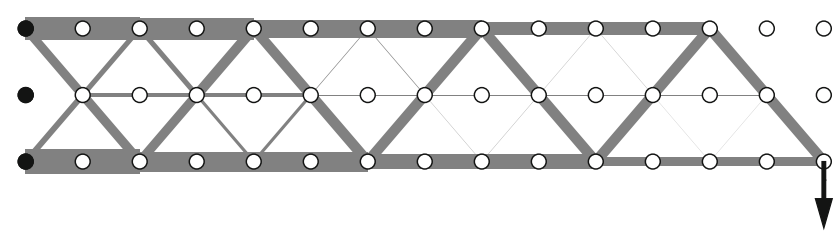

(b) $\gamma=0.8$.

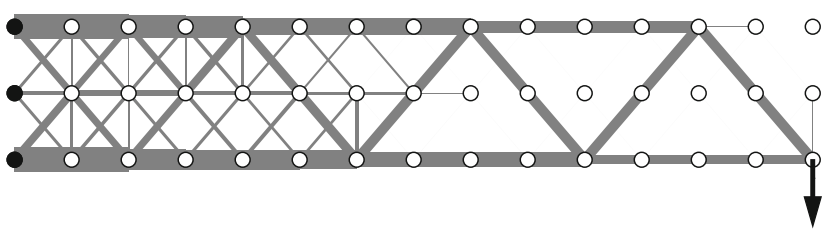

(c) $\gamma=0.9$

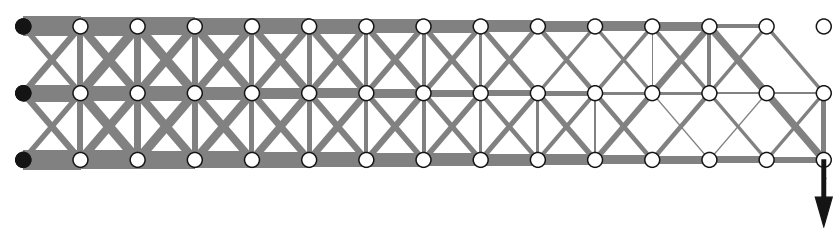

(d) $\gamma=0.999$

Fig. 5 Optimal structures for Example I with partial degradation of one member. The amount of degradation (reduction of member area) is measured by the relative damage factor $\gamma$. Compare with the optimal nominal design in Fig. $3 \mathrm{a}$ and the optimal fail-safe design for complete damage in Fig. 4a

of the size of the working set, and thus the size of the relaxation. Although the investigation is not particularly extensive and the timings are not completely revealing, the $m$ value is set to 40 in the rest of the numerical examples.

\subsection{Discussions}

The final working sets $\mathcal{W}$ presented in Tables 2, 3, and 4 only contain relatively few inactive constraints for the presented examples and the chosen parameter $m$. This is despite the very simple working-set update procedure and the rather arbitrary choice of $m$.

The working-set approach is generally faster than solving the problem $\left(\mathrm{P}_{\mathrm{SDP}}\right)$ including all the damage scenarios. One example illustrating this is Example I Complete $(1,-)$. For this problem instance, the working set requires $145 \mathrm{~s}$ as reported in Table 2, whereas solving the full problem requires over $500 \mathrm{~s}$ (not reported in any of the tables). The difference is perhaps not substantial for this problem 
Table 2 Numerical results with the working-set approach for fail-safe design of Example I

\begin{tabular}{|c|c|c|c|c|c|c|c|}
\hline Damage (bars, $\gamma$ ) & $m$ & Itn. & $|\mathcal{W}|$ & $|\mathcal{A}|$ & CPU (s) & Compliance & $\tau$ \\
\hline $\operatorname{Nominal}(-,-)$ & - & - & - & - & 3.2 & 3.31682 & - \\
\hline Complete $(1,-)$ & 1 & 25 & 24 & 21 & 570.2 & 4.54726 & 8.06481 \\
\hline Complete $(1,-)$ & 5 & 7 & 28 & 21 & 230.1 & 4.54726 & 8.06481 \\
\hline Complete $(1,-)$ & 10 & 4 & 27 & 21 & 145.0 & 4.54726 & 8.06481 \\
\hline Complete $(1,-)$ & 20 & 3 & 28 & 20 & 100.8 & 4.54726 & 8.06481 \\
\hline Complete $(1,-)$ & 40 & 2 & 40 & 21 & 96.1 & 4.54726 & 8.06481 \\
\hline Complete $(1,-)$ & 80 & 2 & 40 & 21 & 102.2 & 4.54726 & 8.06481 \\
\hline Degradation $(1,0.5)$ & 40 & 2 & 40 & 6 & 62.2 & 3.32456 & 3.47884 \\
\hline Degradation $(1,0.6)$ & 40 & 2 & 40 & 6 & 67.4 & 3.32456 & 3.55598 \\
\hline Degradation $(1,0.7)$ & 40 & 2 & 40 & 6 & 91.0 & 3.32456 & 3.68456 \\
\hline Degradation $(1,0.8)$ & 40 & 2 & 40 & 6 & 96.1 & 3.36390 & 3.93108 \\
\hline Degradation $(1,0.9)$ & 40 & 2 & 40 & 10 & 82.5 & 3.43936 & 4.56411 \\
\hline Degradation $(1,0.99)$ & 40 & 2 & 40 & 19 & 76.6 & 4.22084 & 7.42916 \\
\hline Degradation $(1,0.999)$ & 40 & 2 & 40 & 19 & 74.0 & 4.51228 & 7.99977 \\
\hline Degradation $(2,0.5)$ & 40 & 2 & 40 & 15 & 164.9 & 3.32456 & 3.63313 \\
\hline Degradation $(2,0.6)$ & 40 & 3 & 45 & 5 & 192.4 & 3.33285 & 3.78651 \\
\hline Degradation $(2,0.7)$ & 40 & 2 & 40 & 13 & 121.2 & 3.37151 & 4.02828 \\
\hline Degradation $(2,0.8)$ & 40 & 2 & 40 & 17 & 98.8 & 3.41315 & 4.47771 \\
\hline Degradation $(2,0.9)$ & 40 & 2 & 40 & 12 & 129.0 & 3.46231 & 5.77739 \\
\hline Degradation $(2,0.99)$ & 40 & 5 & 131 & 25 & 942.2 & 5.33166 & 14.9118 \\
\hline Degradation $(2,0.999)$ & 40 & 6 & 146 & 31 & 1593.9 & 6.53094 & 17.2946 \\
\hline
\end{tabular}

but becomes more pronounced as the number of damage scenarios increases.

The optimal objective function values for the relevant degradation problems seemingly approach the objective value obtained for the corresponding complete damage problems as the relative damage parameter approaches one. This holds for example for Example I Degradation $(1,0.999)$ which has an objective function very close to the one reported for Example I Complete $(1,-)$. The same observation can be made regarding Example II Degradation $(1,0.999)$ and Complete $(1,-)$ as well as Example II Degradation $(2,0.999)$ and Complete $(2,-)$
Table 3 Numerical results with the working-set approach for fail-safe design of Example II

\begin{tabular}{|c|c|c|c|c|c|c|c|}
\hline Damage (bars, $\gamma$ ) & $m$ & Itn. & $|\mathcal{W}|$ & $|\mathcal{A}|$ & CPU (s) & Compliance & $\tau$ \\
\hline Nominal $(-,-)$ & - & - & - & - & 1.5 & 0.13621 & - \\
\hline Complete $(1,-)$ & 40 & 3 & 8 & 6 & 2.6 & 0.26036 & 0.395852 \\
\hline Degradation $(1,0.5)$ & 40 & 2 & 6 & 6 & 2.2 & 0.13621 & 0.158913 \\
\hline Degradation $(1,0.6)$ & 40 & 2 & 6 & 6 & 1.3 & 0.13621 & 0.170264 \\
\hline Degradation $(1,0.7)$ & 40 & 2 & 6 & 6 & 1.6 & 0.13621 & 0.189182 \\
\hline Degradation $(1,0.8)$ & 40 & 2 & 6 & 6 & 1.5 & 0.13621 & 0.227019 \\
\hline Degradation $(1,0.9)$ & 40 & 3 & 10 & 6 & 2.4 & 0.21545 & 0.304376 \\
\hline Degradation $(1,0.99)$ & 40 & 3 & 8 & 6 & 2.3 & 0.25241 & 0.386873 \\
\hline Degradation $(1,0.999)$ & 40 & 3 & 8 & 6 & 2.2 & 0.25956 & 0.394982 \\
\hline Complete $(2,-)$ & 40 & 3 & 48 & 8 & 6.0 & 0.34349 & 1.25461 \\
\hline Degradation $(2,0.5)$ & 40 & 2 & 40 & 40 & 2.6 & 0.13621 & 0.181615 \\
\hline Degradation $(2,0.6)$ & 40 & 2 & 40 & 40 & 2.7 & 0.13621 & 0.204317 \\
\hline Degradation $(2,0.7)$ & 40 & 2 & 40 & 40 & 2.8 & 0.13621 & 0.242153 \\
\hline Degradation $(2,0.8)$ & 40 & 3 & 44 & 44 & 8.5 & 0.16776 & 0.308501 \\
\hline Degradation $(2,0.9)$ & 40 & 3 & 48 & 8 & 9.0 & 0.19736 & 0.435571 \\
\hline Degradation $(2,0.99)$ & 40 & 3 & 56 & 8 & 8.7 & 0.32070 & 0.887377 \\
\hline Degradation $(2,0.999)$ & 40 & 3 & 48 & 8 & 7.5 & 0.33794 & 1.240550 \\
\hline
\end{tabular}


Table 4 Numerical results with the working-set approach for fail-safe design of Example III

\begin{tabular}{llllllll}
\hline Damage (Bars, $\gamma)$ & $m$ & Itn. & $|\mathcal{W}|$ & $|\mathcal{A}|$ & CPU (s) & Compliance & $\tau$ \\
\hline Nominal (-,-) & - & - & - & - & 4.7 & 4.43051 & - \\
Complete (1,-) & 40 & 2 & 40 & 18 & 193.4 & 5.51354 & 6.86492 \\
Degradation (1,0.5) & 40 & 2 & 40 & 40 & 176.9 & 4.43392 & 4.79609 \\
Degradation (1,0.6) & 40 & 2 & 40 & 40 & 170.2 & 4.61006 & 4.89173 \\
Degradation (1,0.7) & 40 & 2 & 40 & 40 & 181.9 & 4.61541 & 5.03722 \\
Degradation (1,0.8) & 40 & 2 & 40 & 13 & 163.7 & 4.66267 & 5.30954 \\
Degradation (1,0.9) & 40 & 2 & 40 & 21 & 153.3 & 5.06919 & 5.80888 \\
Degradation (1,0.99) & 40 & 2 & 40 & 18 & 219.5 & 5.36964 & 6.72907 \\
Degradation (1,0.999) & 40 & 2 & 40 & 18 & 196.9 & 5.49928 & 6.85213 \\
Degradation (2,0.5) & 40 & 4 & 100 & 100 & 1057.7 & 4.60248 & 4.98907 \\
Degradation (2,0.6) & 40 & 5 & 142 & 142 & 2236.3 & 4.63591 & 5.16425 \\
Degradation (2,0.7) & 40 & 6 & 163 & 22 & 3599.5 & 4.72459 & 5.43005 \\
Degradation (2,0.8) & 40 & 11 & 273 & 29 & 13150.6 & 4.86825 & 5.87880 \\
Degradation (2,0.9) & 40 & 6 & 200 & 24 & 4356.42 & 5.2031 & 6.62705 \\
\hline
\end{tabular}

The results in Tables 2 and 3 indicate that only few of the stiffness constraints are included in the final working set for the optimal fail-safe designs. This could be partially caused by the relatively low number of bars in the ground structures. This should be investigated further but is left for future research.

\subsection{Generalizations}

The primal fail-safe problem $\left(\mathrm{P}_{\mathrm{SDP}}\right)$ can with minor modifications to the problem and the code presented in the appendices be generalized to include robustness to perturbations in the load following the ideas in, e.g., Ben-Tal and Nemirovski (1997). The presented problems can also be generalized to accommodate a constraint on the lowest natural frequency modeled as a matrix inequality (see, e.g., Achtziger and Kočvara (2007)).

Generalizations of the problem formulation and the working-set approach to more advanced structures and engineering relevant design requirements are possible. With few exceptions, convexity is lost and global optimality can therefore not be ensured. The idea of solving a sequence of relaxations instead of including all constraints from the start is likely to work well if the number of active scenarios at the found design is modest. Additionally, one can argue that solving a sequence of relaxations could mitigate some issues with non-convexity. This is, however, speculative and left for future research.

\section{Conclusions}

The working-set approach based on solving a sequence of convex relaxations converges in a few outer iterations. The number of active damage scenarios at the optimal design is modest for the considered examples. The numerical results suggest that the optimal topology can change drastically between the nominal and the fail-safe design even in the situation that only one member is partially degraded. The number of members in the optimal fail-safe topology may increase significantly. This observation is consistent with the results for fail-safe continuum minimum compliance problems presented in, e.g., Jansen et al. (2014). Due to the choices of structure (truss), the associated mechanical restrictions, and the problem formulation the engineering use of the algorithm and the results in this article are of course limited. The main use of the optimal designs is as benchmarks for other algorithms for optimal fail-safe design. Due to the convexity properties, global optimality of the solutions can be ensured, which is an important property for benchmarks in optimization.

\section{Replication of results}

The numerical results presented in Section 6 can all be reproduced. The implementation of the working-set approach used to generate the results is in accordance with Algorithm 1 and the details presented in Section 5. The main parts of the CVX code used to solve the optimal design problems are included in Appendix A for the nominal problems and in Appendix B for the fail-safe problems. The dimensions of the design domains, the type and position of the boundary conditions, the details of the ground structures, and the external loads are all stated in Section 6. The routines for computing the element stiffness matrices for the truss members follow the descriptions presented in e.g. Cook et al. (2002). 
Acknowledgments The author would like to sincerely thank two anonymous reviewers for their knowledgeable comments and suggestions that significantly improved the manuscript in terms of contents and presentation.

Funding information The research presented in this manuscript is funded by Independent Research Fund Denmark through the research project Fail-Safe Structural Optimization (SELMA) with grant no. 7017-00084B.

\section{Compliance with ethical standards}

Conflict of interest The author declares no conflict of interest.

\section{Appendix A: Matlab codes for the nominal problems}

The CVX code for modeling and solving the nominal single-load minimum compliance problem $\left(\mathrm{P}_{\mathrm{N}}\right)$ modeled as a SDP is given below. The code has been shortened for readability by removing the dual variables and reducing the problem to a single load.

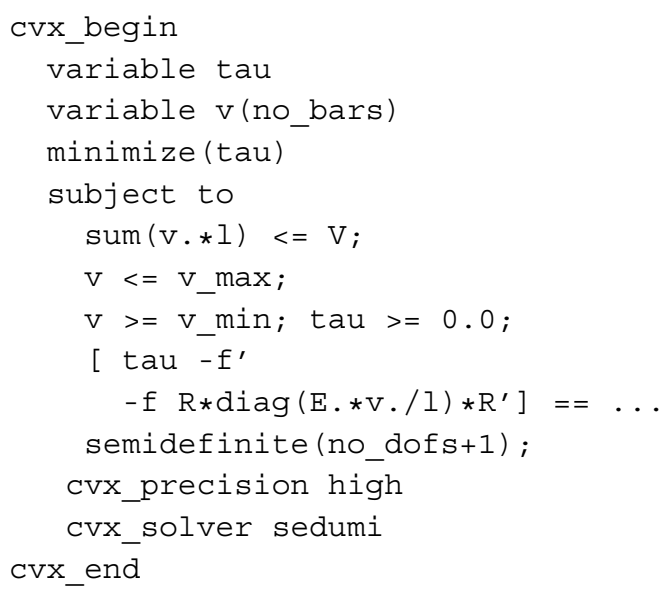

The corresponding CVX commands for modeling and solving the nominal minimum compliance problem $\left(\mathrm{P}_{\mathrm{N}}\right)$ as the Second Order Cone Program is stated below. The code has again been shortened for brevity and clarity.

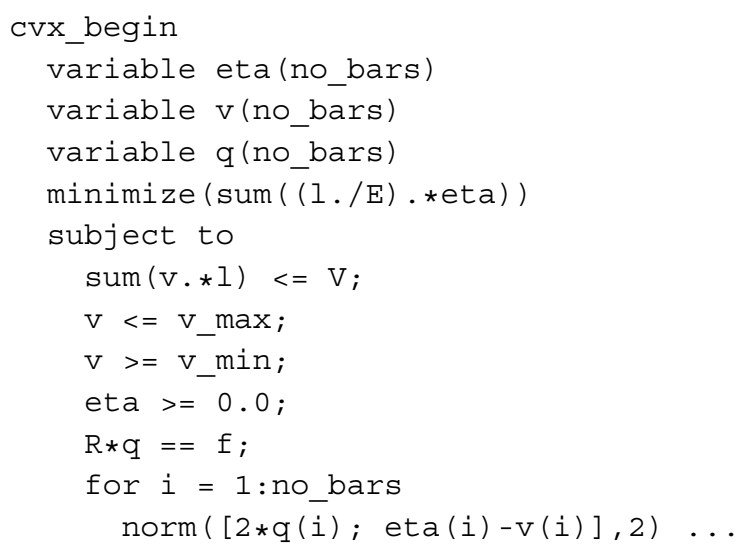

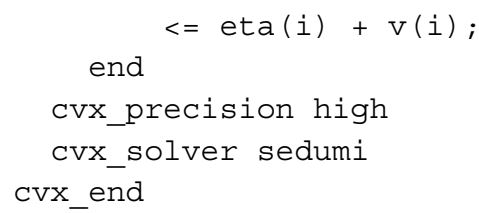

\section{Appendix B: Matlab codes for the fail-safe problems}

The CVX code for modeling and solving the fail-safe minimum compliance problem $\left(\mathrm{P}_{\mathrm{SDP}}\right)$ modeled as an SDP is given below. The code explicitly includes one linear matrix inequality per damage scenario. The functionality of the code as listed only supports single-load problems. Generalization to multiple loads is possible. The matrix $\mathrm{C}$ contains, in each row, one combination of damaged bars.

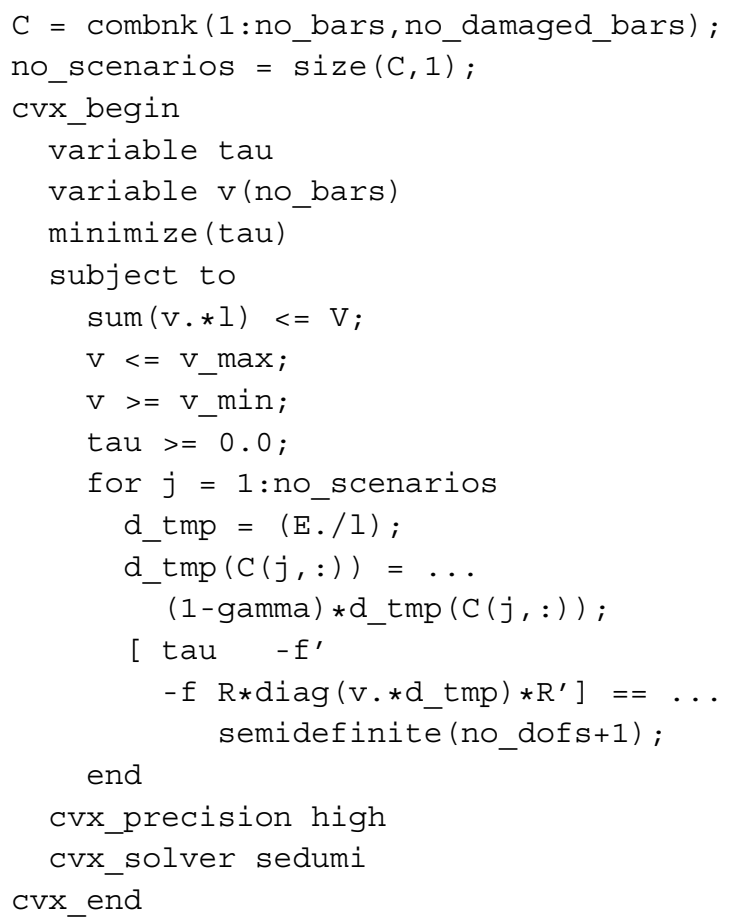

The code above is slightly rewritten to accommodate for the working-set approach in Algorithm 1. The relevant part of the code used to solve the relaxation $\left(\mathrm{P}_{\mathrm{SDP}}^{k}\right)$ is stated below. The working set is stored in a vector $\mathrm{W}$ in which each entry points to the relevant row in the matrix $C$.

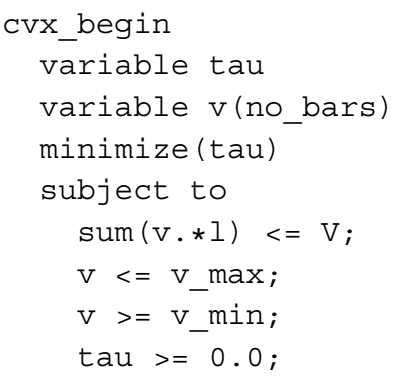




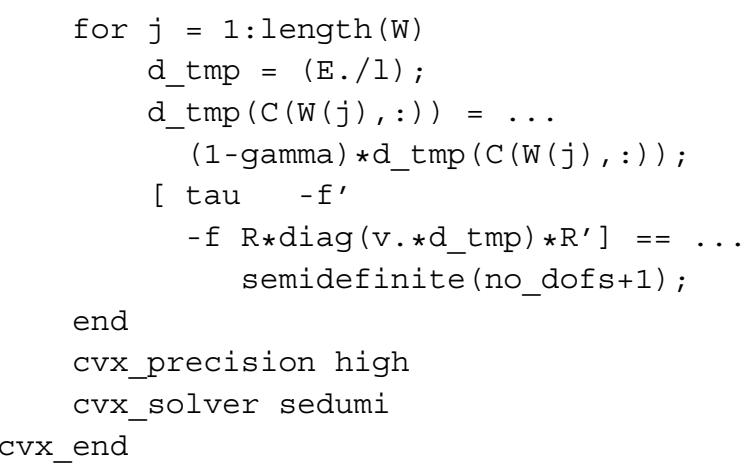

\section{References}

Achtziger W (1996) Truss topology optimization including bar properties different for tension and compression. Structural Optimization 12(1):63-74

Achtziger W (1998) Multiple-load truss topology and sizing optimization: some properties of minimax compliance. J Optim Theory Appl 98(2):255-280

Achtziger W, Bendsøe M (1999) Optimal topology design of discrete structures resisting degradation effects. Structural Optimization 17(1):74-78

Achtziger W, Kanzow C (2008) Mathematical programs with vanishing constraints: optimality conditions and constraint qualifications. Math Program 114:69-99

Achtziger W, Kočvara M (2007) Structural topology optimization with eigenvalues. SIAM J Optim 18(4):1129-1164

Achtziger W, Bendsøe M, Ben-Tal A, Zowe J (1992) Equivalent displacement based formulations for maximum strength truss topology design. Impact of Computing in Science and Engineering 4(4):315-345

Ben-Tal A, Nemirovski A (1997) Robust truss topology design via semidefinite programming. SIAM J Optim 7(4):991-1016

Ben-Tal A, Nemirovski A (2001) Lectures on modern convex optimization: analysis, algorithms and engineering applications. SIAM, Philadelphia

Bendsøe M, Sigmund O (2003) Topology optimization - theory, methods, and applications. Springer Verlag

Boyd S, Vandenberghe L (2004) Convex optimization. Cambridge University Press, Cambridge

Cheng G, Guo X (1997) $\varepsilon$-relaxed approach in structural topology optimization. Structural Optimization 13(4):258-266

Cook RD, Malkus DS, Plesha ME, Witt RJ (2002) Concepts and applications of finite element analysis, 4th edn. Wiley, New York

Dorn W, Gomory R, Greenberg H (1964) Automatic design of optimal structures. J de Mécanique 3:25-52

Fujisawa K, Kojima M, Nakata K (1997) Exploiting sparsity in primaldual interior-point methods for semidefinite programming. Math Program 79(1-3):235-253

Gilbert M, Tyas A (2003) Layout optimization of large-scale pinjointed frames. Eng Comput 20(7-8):1044-1064

Grant M, Boyd S (2008) Graph implementations for nonsmooth convex programs. In: Blondel V, Boyd S, Kimura H (eds) Recent Advances in Learning and Control, Lecture Notes in Control and Information Sciences. Springer-Verlag Limited, pp 95110
Grant M, Boyd S (2014) CVX: Matlab software for disciplined convex programming, version 2.1. http://cvxr.com/cvx

Hemp W (1973) Optimum structures. Clarendon Press

Jansen M, Lombaert G, Schevenels M, Sigmund O (2014) Topology optimization of fail-safe structures using a simplified local damage model. Struct Multidiscip Optim 49(4):657-666

Kanno Y (2012) Worst scenario detection in limit analysis of trusses against deficiency of structural components. Eng Struct 42:33-42

Kanno Y (2016) Global optimization of trusses with constraints on number of different cross-sections: a mixed-integer second-order cone programming approach. Comput Optim Appl 63(1):203236

Kanno Y (2017) Redundancy optimization of finite-dimensional structures: concept and derivative-free algorithm. J Struct Eng 143(1):04016,151

Kanno Y, Ben-Haim Y (2011) Redundancy and robustness, or when is redundancy redundant? J Struct Eng 137(9):935-945

Kirsch U (1990) On singular topologies in optimum structural design. Structural Optimization 2(3):133-142

Kreisselmeier G, Steinhauser R (1983) Application of vector performance optimization to a robust control loop design for a fighter aircraft. Int J Control 37(2):251-284

Lobo M, Vandenberghe L, Boyd S, Lebret H (1998) Applications of second-order cone programming. Linear Algebra Appl 284(13): $193-228$

Mohr D, Stein I, Matzies T, Knapek C (2014) Redundant robust topology optimization of truss. Optim Eng 15(4):945-972

Rozvany G (1996) Difficulties in truss topology optimization with stress, local buckling and system stability constraints. Structural Optimization 11:213-217

Rozvany G (2001) On design-dependent constraints and topologies. Struct Multidiscip Optim 21:164-172

Sturm J (1999) Using SeDuMi 1.02, a MATLAB toolbox for optimization over symmetric cones. Optim Methods Softw 11-2(14):625-653

Sturm J (2002) Implementation of interior point methods for mixed semidefinite and second order cone optimization problems. Optim Methods Softw 17(6):1105-1154

Sun P, Arora J, Haug E (1976) Fail-safe optimal design of structures. Eng Optim 2(1):43-53

Svanberg K (1987) The method of moving asymptotes - a new method for structural optimization. Int $\mathbf{J}$ Numer Methods Eng 24(2):359-373

Sved G, Ginos Z (1968) Structural optimization under multiple loading. Int J Mech Sci 10(10):803-805

The Mathworks Inc (2018) MATLAB R2018a. Natick, Massachusetts

Toh K, Todd M, Tütüncü R (1999) SDPT3 - A MATLAB software package for semidefinite programming, version 1.3. Optim Methods Softw 11(1-4):545-581

Tütüncü R, Toh K, Todd M (2003) Solving semidefinite-quadraticlinear programs using SDPT3. Math Program 95(2):189-217

Verbart A, Stolpe M (2018) A working-set approach for sizing optimization of frame-structures subjected to time-dependent constraints. Struct Multidiscip Optim 58(4):1367-1382

Yamashita M, Fujisawa K (2003) Implementation and evaluation of SDPA 6.0 (SemiDefinite Programming Algorithm 6.0). Optim Methods Softw 18(4):491-505

Zhou M, Fleury R (2016) Fail-safe topology optimization. Struct Multidiscip Optim 54(5):1225-1243

Publisher's note Springer Nature remains neutral with regard to jurisdictional claims in published maps and institutional affiliations. 